\title{
Hilfer fractional differential inclusions with Erdélyi-Kober fractional integral boundary condition
}

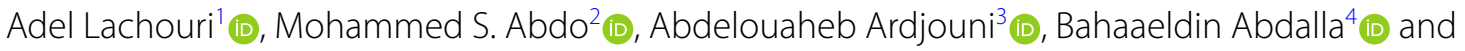 \\ Thabet Abdeljawad ${ }^{4,5,6^{*}}$ (D)
}

\author{
"Correspondence: \\ tabdeljawad@psu.edu.sa \\ ${ }^{4}$ Department of Mathematics and \\ General Sciences, Prince Sultan \\ University, Riyadh, Saudi Arabia \\ ${ }^{5}$ Department of Medical Research, \\ China Medical University, Taichung \\ 40402, Taiwan \\ Full list of author information is \\ available at the end of the article
}

\begin{abstract}
In this article, we debate the existence of solutions for a nonlinear Hilfer fractional differential inclusion with nonlocal Erdélyi-Kober fractional integral boundary conditions (FIBC). Both cases of convex- and nonconvex-valued right-hand side are considered. Our obtained results are new in the framework of Hilfer fractional derivative and Erdélyi-Kober fractional integral with FIBC via the fixed point theorems (FPTs) for a set-valued analysis. Some pertinent examples demonstrating the effectiveness of the theoretical results are presented.
\end{abstract}

MSC: 34A08; 34A12; 34B15

Keywords: Fractional inclusion problem; Hilfer and Erdélyi-Kober fractional operators; Existence; Fractional integral boundary conditions; Convex; Nonconvex; Fixed point theorems

\section{Introduction}

In recent years, fractional differential equation (FDE) theory has received very broad attention in the fields of both pure and applied mathematics, see [27, 35, 39, 45]. FDEs and fractional differential inclusions (FDIs) emerge naturally in diverse areas of science with many applications, see, e.g., $[19,23,25,26,28,31,41,47,50]$. In the literature, there are many definitions of fractional derivatives (FDs) and fractional integrals (FIs), e.g., Riemann-Liouville [35], Caputo [21], Hadamard [30], Hilfer [31], Katugampola [34], Caputo-Hadamard [32], Caputo-Katugampola [15], Caputo-Fabrizio [22], AtanganaBaleanu-Caputo [18]. In addition, several new operators have emerged to combine a wide category of FDs, such as $\psi$-Caputo [14] and $\psi$-Hilfer [46].

In the last few years, many researchers have started to discuss the qualitative properties of solutions of fractional FDEs and FDIs, such as existence, uniqueness, stability, controllability, and optimization, etc., see [1-3, 7-10, 20, 43, 44, 49]. Some other researchers have devoted their works to discussing further analytical properties of solutions of such equations and inclusions, while others already have oriented their investigations towards numerical applications and solutions. For further specialized articles on

(c) The Author(s) 2021. This article is licensed under a Creative Commons Attribution 4.0 International License, which permits use sharing, adaptation, distribution and reproduction in any medium or format, as long as you give appropriate credit to the original author(s) and the source, provide a link to the Creative Commons licence, and indicate if changes were made. The images or other third party material in this article are included in the article's Creative Commons licence, unless indicated otherwise in a credit line to the material. If material is not included in the article's Creative Commons licence and your intended use is not permitted by statutory regulation or exceeds the permitted use, you will need to obtain permission directly from the copyright holder. To view a copy of this licence, visit http://creativecommons.org/licenses/by/4.0/. 
the existence, uniqueness, and stability of FDEs involving various types of FDs, we refer to $[4,6,11,16,33,37,38,46]$.

Recently, Asawasamrit et al. [17] have initiated the study of Hilfer FDEs with nonlocal IBCs of the type

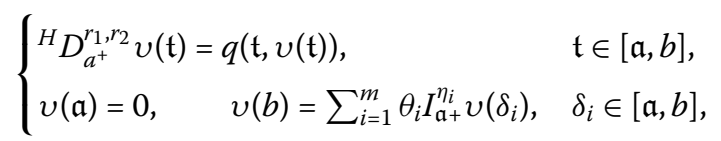

where $1<r_{1}<2,0 \leq r_{2} \leq 1, \eta_{i}>0, \theta_{i} \in \mathbb{R},{ }^{H} D_{a^{+}}^{r_{1}, r_{2}}$ is the Hilfer FD of order $r_{1}$ and type $r_{2}$, $I_{\mathfrak{a}+}^{\eta_{i}}$ is the Riemann-Liouville FI of order $\eta_{i}$. Later, the authors of [42] have investigated the existence and stability results of an implicit problem for FDE (1.1) involving $\psi$-Hilfer FD.

On the other hand, Abbas [5] investigated the existence and Ulam-Hyers stability results for the FDE of type (1.1) with the consideration of Erdélyi-Kober FI instead of RiemannLiouville FI. The set-valued case of problem (1.1) has been studied by Wongcharoen et al. [48].

In order to enhance the work and fill the gap on BVPs of fractional order involving more IBCs, we consider a nonlinear Hilfer-type FDI with Erdélyi-Kober fractional IBC, that is,

$$
\left\{\begin{array}{l}
{ }^{H} D^{r_{1}, r_{2}} v(\mathfrak{t}) \in Q(\mathfrak{t}, v(\mathfrak{t})), \quad \mathfrak{t} \in(0, \mathcal{T}), \mathcal{T}>0 \\
v(0)=0, \quad v(\mathcal{T})=\sum_{i=1}^{m} \theta_{i} I_{\gamma_{i}} \xi_{i} \xi_{i} v\left(\delta_{i}\right)
\end{array}\right.
$$

where ${ }^{H} D^{r_{1}, r_{2}}$ is the Hilfer FD of order $r_{1} \in(1,2)$ and type $r_{2} \in[0,1], I^{\eta_{i} ; \xi_{i}}$ is the ErdélyiKober FI of order $\xi_{i}>0$ with $\gamma_{i}>0$ and $\eta_{i} \in \mathbb{R}, Q:[0, \mathcal{T}] \times \mathbb{R} \rightarrow \mathcal{O}(\mathbb{R})$ is a set-valued map $(\mathrm{svm})$ from $[0, \mathcal{T}] \times \mathbb{R}$ to the family of $\mathcal{O}(\mathbb{R}) \subset \mathbb{R}, \theta_{i} \in \mathbb{R}, \delta_{i} \in(0, T), i=1,2, \ldots, m$. Our main concern in this manuscript is to obtain the existence results for the Hilfer inclusion problem (1.2) involving convex, nonconvex set-valued maps via some FPTs of Leray-Schauder type, as well as those of Covitz and Nadler, where some pertinent examples are built for the demonstration of our findings.

\section{Remark 1}

i) If Erdélyi-Kober FI $I^{\eta_{i} \xi_{i}}$ is replaced by Riemann-Liouville FI $I_{\mathfrak{a}+}^{\eta_{i}}$ in problem (1.2), then the inclusion problem for it has been studied by Wongcharoen et al. [48].

ii) If $r_{2}=0$ in (1.2), then our problem reduces to Riemann-Liouville inclusion problem considered by Ahmad and Ntouyas in [12].

iii) If $r_{2}=1$ in (1.2), then our problem also covers other problems, including those of Caputo type.

This paper is structured as follows. In Sect. 2, we give some fundamental concepts of fractional calculus, set-valued analysis, and FP techniques. In Sect. 3, we study some existence results for the Hilfer inclusion problem (1.2) relying on some FPTs of LeraySchauder, Covitz, and Nadler. At the end, some examples are given in Sect. 4.

\section{Preliminaries}

\subsection{Fractional Calculus (FC)}

In this part, we give some essential ideas of $\mathrm{FC}$ and axiom outcomes that are prerequisites in our analysis. 
Let $\mathfrak{P}=[0, \mathcal{T}], r_{1} \in(1,2), r_{2} \in[0,1]$, where $\mathfrak{v}=r_{1}+r_{2}\left(2-r_{1}\right) \in(1,2]$. By $\mathcal{C}=C(\mathfrak{P}, \mathbb{R})$ we denote the Banach space of all continuous functions $\varkappa: \mathfrak{P} \rightarrow \mathbb{R}$ with norm

$$
\|\varkappa\|=\sup \{|\varkappa(\mathfrak{t})|: \mathfrak{t} \in \mathfrak{P}\}
$$

and $L^{1}(\mathfrak{P}, \mathbb{R})$ is the Banach space of Lebesgue integrable functions $\varkappa: \mathfrak{P} \rightarrow \mathbb{R}$ with norm

$$
\|\varkappa\|_{L^{1}}=\int_{\mathfrak{P}}|\varkappa(\mathfrak{t})| d \mathfrak{t}
$$

Definition 1 ([35]) The Riemann-Liouville FI of a function $\varkappa$ of order $r_{1}$ is described by

$$
I^{r_{1}} \varkappa(\mathfrak{t})=\frac{1}{\Gamma\left(r_{1}\right)} \int_{0}^{\mathfrak{t}}(\mathfrak{t}-\varsigma)^{r_{1}-1} \varkappa(\varsigma) d \varsigma
$$

provided the integral exists.

Definition 2 ([35]) The Riemann-Liouville FD of a function $h$ of order $r_{1}$ is described by

$$
D^{r_{1}} \varkappa(\mathfrak{t})=\left(\frac{d}{d \mathfrak{t}}\right)^{n} I^{\left(n-r_{1}\right)} \varkappa(\mathfrak{t})
$$

where $n=\left[r_{1}\right]+1, n \in \mathbb{N}$.

Definition 3 ([46]) The Hilfer FD of a function $\varkappa$ of order $r_{1}$ and type $r_{2}$ is described by

$$
{ }^{H} D^{r_{1}, r_{2}} \varkappa(\mathfrak{t})=I^{r_{2}\left(n-r_{1}\right)} D^{[n]} I^{\left(1-r_{2}\right)\left(n-r_{1}\right)} \varkappa(\mathfrak{t})
$$

where $D^{[n]}=\left(\frac{d}{d t}\right)^{n}$.

Definition 4 ([35]) The Erdélyi-Kober FI of a function $\varkappa$ of order $\xi>0$ with $\gamma>0$ and $\eta \in \mathbb{R}$ is formulated by

$$
I_{\gamma}^{\eta, \xi} \varkappa(\mathfrak{t})=\frac{\gamma t^{-\gamma(\eta+\xi)}}{\Gamma(\xi)} \int_{0}^{\mathfrak{t}}\left(\mathfrak{t}^{\gamma}-\varsigma^{\gamma}\right)^{\xi-1} \varsigma^{\gamma \eta+\gamma-1} \varkappa(\varsigma) d \varsigma,
$$

provided the integral exists.

Remark 2 For $\gamma=1,(2.1)$ is reduced to the Kober operator

$$
K^{\eta, \xi} \varkappa(\mathfrak{t})=\frac{t^{-(\eta+\xi)}}{\Gamma(\xi)} \int_{0}^{\mathfrak{t}}(\mathfrak{t}-\varsigma)^{\xi-1} \varsigma^{\eta} \varkappa(\varsigma) d \varsigma, \quad \xi, \eta>0 .
$$

For $\eta=0,(2.2)$ leads to the Riemann-Liouville FI with a power weight,

$$
K^{0, \xi} \varkappa(\mathfrak{t})=\frac{t^{-\xi}}{\Gamma(\xi)} \int_{0}^{\mathfrak{t}}(\mathfrak{t}-\varsigma)^{\xi-1} \varkappa(\varsigma) d \varsigma, \quad \xi>0 .
$$


Lemma 1 ([35]) Let $\xi, \eta>0$ and $\gamma, \alpha \in \mathbb{R}$. Then

$$
I_{\gamma}^{\eta, \xi} \mathfrak{t}^{\alpha}=\frac{\mathfrak{t}^{\alpha} \Gamma\left(\eta+\frac{\alpha}{\gamma}+1\right)}{\Gamma\left(\eta+\frac{\alpha}{\gamma}+\xi+1\right)} .
$$

Lemma 2 ([35]) Let $\gamma, \xi, \beta>0$. Then

$$
\int_{0}^{\mathfrak{t}}\left(\mathfrak{t}^{\gamma}-\varsigma^{\gamma}\right)^{\xi-1} \varsigma^{\beta-1} d \varsigma=\frac{\mathfrak{t}^{\gamma(\xi-1)+\beta}}{\gamma} B\left(\frac{\beta}{\gamma}, \xi\right),
$$

where $B(\cdot, \cdot)$ is the beta function determined by

$$
B\left(r_{1}, r_{2}\right)=\int_{0}^{1}(1-\varsigma)^{r_{1}-1} \varsigma^{r_{2}-1} d \varsigma \quad\left(\Re\left(r_{1}\right)>0, \Re\left(r_{2}\right)>0\right) .
$$

Lemma 3 ([31]) Let $r_{1} \in(1,2]$. Then

$$
I^{r_{1}} D^{r_{1}} \varkappa(\mathfrak{t})=\varkappa(\mathfrak{t})-\frac{\left(I^{1-r_{1}} \varkappa\right)(0)}{\Gamma\left(r_{1}\right)} t^{r_{1}-1}-\frac{\left(I^{2-r_{1}} \varkappa\right)(0)}{\Gamma\left(r_{1}-1\right)} t^{r_{1}-2} .
$$

Lemma 4 ([5]) Let

$$
\Lambda=\mathcal{T}^{\mathfrak{v}-1}-\sum_{i=1}^{m} \frac{\theta_{i} \delta_{i}^{\mathfrak{v}-1} \Gamma\left(\eta_{i}+\frac{\mathfrak{v}-1}{\gamma_{i}}+1\right)}{\Gamma\left(\eta_{i}+\frac{\mathfrak{v}-1}{\gamma_{i}}+\xi_{i}+1\right)} \neq 0,
$$

and consider any $\mathfrak{h} \in \mathcal{C}$. Then the solution of the nonlocal BVP

$$
\left\{\begin{array}{l}
{ }^{H} D^{r_{1}, r_{2}} v(\mathfrak{t})=\mathfrak{h}(\mathfrak{t}), \quad \mathfrak{t} \in(0, \mathcal{T}), \mathcal{T}>0, \\
v(0)=0, \quad v(\mathcal{T})=\sum_{i=1}^{m} \theta_{i} I_{\gamma_{i}} \eta_{i} \xi_{i} v\left(\delta_{i}\right),
\end{array}\right.
$$

is obtained as

$$
v(\mathfrak{t})=I^{r_{1}} \mathfrak{h}(\mathfrak{t})+\frac{\mathfrak{t}^{\mathfrak{v}-1}}{\Lambda}\left(\sum_{i=1}^{m} \theta_{i} I_{\gamma_{i}}^{\eta_{i} ; \xi_{i}} I^{r_{1}} \mathfrak{h}\left(\delta_{i}\right)-I^{r_{1}} \mathfrak{h}(\mathcal{T})\right) .
$$

\subsection{Set-valued analysis}

We recall some concepts concerning the theory of set-valued maps. For this, let $(v,\|\cdot\|)$ be a Banach space and $\mathfrak{N}: v \rightarrow \mathcal{O}(v)$ be a set-valued map, which

(a) is convex-(closed-)valued if $\mathfrak{N}(v)$ is convex (closed) for any $v \in \mathcal{O}$,

(b) is bounded if $\mathfrak{N}(\mathcal{B})=\bigcup_{v \in \mathcal{B}} \mathfrak{N}(v)$ is bounded in $v$ for all bounded sets $\mathcal{B}$ of $v$, i.e.,

$$
\sup _{v \in \mathcal{B}}\{\{\sup |\rho|: \rho \in \mathfrak{N}(v)\}\}<\infty
$$

(c) is measurable if $\forall \rho \in \mathbb{R}$, the function

$$
v \rightarrow d(\rho, \mathfrak{N}(v))=\inf \{|\rho-\eta|: \eta \in \mathfrak{N}(v)\},
$$

is measurable. 
For the definitions of completely continuous and u.s.c., we refer to [13].

Moreover, a collection of selections of $Q$ at a point $\rho \in \mathcal{C}$ is defined by

$$
\mathcal{R}_{Q, \rho}=\left\{\tilde{v} \in L^{1}(\mathfrak{P}, \mathbb{R}): \tilde{v}(\mathfrak{t}) \in Q(\mathfrak{t}, \rho) \text { for a.e. } \mathfrak{t} \in \mathfrak{P}\right\} .
$$

Next, we denote

$$
\mathcal{O}_{k}(v)=\{\mathcal{N} \in \mathcal{O}(v): \mathcal{N} \text { is nonempty and has property } k\}
$$

where $\mathcal{O}_{\text {cp }}, \mathcal{O}_{b}, \mathcal{O}_{\mathrm{cl}}, \mathcal{O}_{c}$ denote the categories of all compact, bounded, closed, and convex subsets of $v$, respectively. Also, $\mathcal{O}_{\mathrm{cp}, c}$ denotes the category of all convex and compact subsets of $v$.

Definition 5 ([26]) A set-valued map $Q: \mathfrak{P} \times \mathbb{R} \rightarrow \mathcal{O}(\mathbb{R})$ is called Carathéodory whenever the map $\mathfrak{t} \rightarrow Q(\mathfrak{t}, v)$ is measurable for any $v \in \mathbb{R}$, and the map $v \rightarrow Q(\mathfrak{t}, v)$ is u.s.c. for (a.e.) all $\mathfrak{t} \in \mathfrak{P}$.

Moreover, a set-valued map $Q$ is called $L^{1}$-Carathéodory if $\forall w>0$, there exists $\varphi \in$ $L^{1}\left(\mathfrak{P}, \mathbb{R}^{+}\right)$such that

$$
\|Q(\mathfrak{t}, v)\|=\sup \{|\tilde{v}|: \tilde{v} \in Q(\tilde{v}, v)\} \leq \varphi(\mathfrak{t})
$$

for all $\|v\| \leq w$ and for a.e. $\mathfrak{t} \in \mathfrak{P}$.

We will use the following lemmas that will play an important role in the achievement of the desired results in this research.

Lemma 5 ([26, Proposition 1.2]) Let $\operatorname{Gr}(\mathfrak{N})=\{(v, \rho) \in v \times Z, \rho \in \mathfrak{N}(v)\}$ be the graph of $\mathfrak{N}$. If $\mathfrak{N}: v \rightarrow \mathcal{O}_{\mathrm{cl}}(v)$ is u.s.c., then $\operatorname{Gr}(\mathfrak{N})$ is a closed subset of $v \times Z$. If $\mathfrak{N}$ is completely continuous and has a closed graph, then it is u.s.c.

Lemma $6([40])$ Let $v$ be a separable Banach space, $Q: \mathfrak{P} \times v \rightarrow \mathcal{O}_{\mathrm{cp}, c}(v)$ be an $L^{1}$ Carathéodory set-valued map, and $\mathcal{Z}: L^{1}(\mathfrak{P}, v) \rightarrow C(\mathfrak{P}, v)$ be a linear continuous mapping. Then the operator

$$
\mathcal{Z} \circ \mathcal{R}_{Q}: C(\mathfrak{P}, v) \rightarrow \mathcal{O}_{\mathrm{cp}, c}(C(\mathfrak{P}, v)), \quad v \rightarrow\left(\mathcal{Z} \circ \mathcal{R}_{Q}\right)(v)=\mathcal{Z}\left(\mathcal{R}_{Q, v}\right)
$$

has a closed graph in $C(\mathfrak{P}, v) \times C(\mathfrak{P}, v)$.

\section{Existence results for (1.2)}

Definition 6 A function $v \in \mathcal{C}$ is considered a solution of (1.2), if there is an integrable function $\tilde{v} \in L^{1}(\mathfrak{P}, \mathbb{R})$ with $\tilde{v}(\mathfrak{t}) \in Q(\mathfrak{t}, v)$ for all $\mathfrak{t} \in \mathfrak{P}$ satisfying the nonlocal fractional IBC

$$
v(0)=0, \quad v(\mathcal{T})=\sum_{i=1}^{m} \theta_{i} I_{\gamma_{i}}^{\eta_{i} ; \xi_{i}} v\left(\delta_{i}\right)
$$


and

$$
\begin{aligned}
v(\mathfrak{t})= & I^{r_{1}} \tilde{v}(\mathfrak{t})+\frac{\mathfrak{t}^{\mathfrak{v}-1}}{\Lambda}\left(\sum_{i=1}^{m} \theta_{i} I_{\gamma_{i}}^{\eta_{i} \xi_{i}} I^{r_{1}} \tilde{v}\left(\delta_{i}\right)-I^{r_{1}} \tilde{v}(\mathcal{T})\right) \\
= & \frac{1}{\Gamma\left(r_{1}\right)} \int_{0}^{\mathfrak{t}}(\mathfrak{t}-\varsigma)^{r_{1}-1} \tilde{v}(\varsigma) d \varsigma \\
& +\frac{\mathfrak{t}^{\mathfrak{v}-1}}{\Lambda}\left(\sum_{i=1}^{m} \theta_{i} \frac{\gamma_{i} \delta_{i}^{-\gamma_{i}\left(\xi_{i}+\eta_{i}\right)}}{\Gamma\left(\xi_{i}\right) \Gamma\left(r_{1}\right)} \int_{0}^{\delta_{i}} \frac{\varsigma^{\gamma_{i}+\eta_{i}+\gamma_{i}-1}}{\left(\delta_{i}^{\gamma_{i}}-\varsigma^{\left.\gamma_{i}\right)}\right.}\left(\int_{0}^{\varsigma}(\varsigma-\sigma)^{r_{1}-1} \tilde{v}(\sigma) d \sigma\right) d \varsigma\right. \\
& \left.-\frac{1}{\Gamma\left(r_{1}\right)} \int_{0}^{\mathcal{T}}(\mathcal{T}-\varsigma)^{r_{1}-1} \tilde{v}(\varsigma) d \varsigma\right) .
\end{aligned}
$$

\subsection{The U.S.C. case}

The first result deals with a convex-valued $Q$ relying on Leray-Schauder principle for setvalued maps [29].

\section{Theorem 1 Let}

$$
\varrho=\frac{1}{\Gamma\left(r_{1}+1\right)}\left(\mathcal{T}^{r_{1}}+\frac{\mathcal{T}^{\mathfrak{v}+r_{1}-1}}{|\Lambda|}+\frac{\mathcal{T}^{\mathfrak{v}-1}}{|\Lambda|}\left(\sum_{i=1}^{m}\left|\theta_{i}\right| \frac{\delta_{i}^{r_{1}} \Gamma\left(\eta_{i}+\frac{r_{1}}{\gamma_{i}}+1\right)}{\Gamma\left(\eta_{i}+\frac{r_{1}}{\gamma_{i}}+\xi_{i}+1\right)}\right)\right)
$$

and assume that:

$\left(\right.$ As1) $Q: \mathfrak{P} \times \mathbb{R} \rightarrow \mathcal{O}_{\mathrm{cp}, c}(\mathbb{R})$ is an $L^{1}$-Carathéodory set-valued map,

(As2) There is a nondecreasing function $\vartheta \in C\left(\mathbb{R}^{+}, \mathbb{R}^{+}\right)$and a continuous function $P: \mathfrak{P} \rightarrow \mathbb{R}^{+}$such that

$$
\|Q(\mathfrak{t}, v)\|_{\mathcal{O}}=\sup \{|\rho|: \rho \in Q(\mathfrak{t}, v)\} \leq P(\mathfrak{t}) \vartheta(\|v\|), \quad \forall(\mathfrak{t}, v) \in \mathfrak{P} \times \mathbb{R}
$$

(As3) There is a constant $\mathcal{L}>0$ such that

$$
\frac{\mathcal{L}}{\varrho\|P\| \vartheta(\mathcal{L})}>1
$$

Then problem (1.2) has at least one solution on $\mathfrak{P}$.

Proof Initially, to write problem (1.2) as an FP problem, we consider the operator $\tilde{\mathcal{S}}: \mathcal{C} \rightarrow$ $\mathcal{O}(\mathcal{C})$ defined by

$$
\tilde{\mathcal{S}}(v)=\left\{\phi \in \mathcal{C}: \phi(\mathfrak{t})=\left\{\begin{array}{c}
\frac{1}{\Gamma\left(r_{1}\right)} \int_{0}^{\mathfrak{t}}(\mathfrak{t}-\varsigma)^{r_{1}-1} \tilde{v}(\varsigma) d \varsigma \\
+\frac{\mathfrak{t}^{\mathfrak{v}-1}}{\Lambda}\left(\sum_{i=1}^{m} \theta_{i} \frac{\gamma_{i} \delta_{i}^{-\gamma_{i}}\left(\xi_{i}+\eta_{i}\right)}{\Gamma\left(\xi_{i}\right) \Gamma\left(r_{1}\right)}\right. \\
\times \int_{0}^{\delta_{i}} \frac{\varsigma^{\gamma_{i}+\eta_{i}+\gamma_{i}-1}}{\left(\delta_{i}^{\gamma_{i}}-\varsigma^{\left.\gamma_{i}\right)}\right.}\left(\int_{0}^{\varsigma}(\varsigma-\sigma)^{r_{1}-1} \tilde{v}(\sigma) d \sigma\right) d \varsigma \\
\left.-\frac{1}{\Gamma\left(r_{1}\right)} \int_{0}^{\mathcal{T}}(\mathcal{T}-\varsigma)^{r_{1}-1} \tilde{v}(\varsigma) d \varsigma\right),
\end{array}\right\}\right.
$$

for $\tilde{v} \in \mathcal{R}_{Q, v}$. Obviously, the solution of (1.2) is an FP of the operator $\tilde{\mathcal{S}}$. The proof steps will be presented as follows:

Step 1. The set-valued map $\tilde{\mathcal{S}}(v)$ is convex for any $v \in \mathcal{C}$. 
Let $\phi_{1}, \phi_{2} \in \tilde{\mathcal{S}}(v)$. Then, there exist $\tilde{v}_{1}, \tilde{v}_{2} \in \mathcal{R}_{Q, v}$ such that

$$
\begin{aligned}
\phi_{j}(\mathfrak{t})= & \frac{1}{\Gamma\left(r_{1}\right)} \int_{0}^{\mathfrak{t}}(\mathfrak{t}-\varsigma)^{r_{1}-1} \tilde{v}(\varsigma) d \varsigma \\
& +\frac{\mathfrak{t}^{\mathfrak{v}-1}}{\Lambda}\left(\sum_{i=1}^{m} \theta_{i} \frac{\gamma_{i} \delta_{i}^{-\gamma_{i}\left(\xi_{i}+\eta_{i}\right)}}{\Gamma\left(\xi_{i}\right) \Gamma\left(r_{1}\right)} \int_{0}^{\delta_{i}} \frac{\varsigma^{\gamma_{i}+\eta_{i}+\gamma_{i}-1}}{\left(\delta_{i}^{\gamma_{i}}-\varsigma^{\left.\gamma_{i}\right)}\right.}\left(\int_{0}^{\varsigma}(\varsigma-\sigma)^{r_{1}-1} \tilde{v}_{j}(\sigma) d \sigma\right) d \varsigma\right. \\
& \left.-\frac{1}{\Gamma\left(r_{1}\right)} \int_{0}^{\mathcal{T}}(\mathcal{T}-\varsigma)^{r_{1}-1} \tilde{v}_{j}(\varsigma) d \varsigma\right), \quad j=1,2, \forall \mathfrak{t} \in \mathfrak{P} .
\end{aligned}
$$

Let $\lambda \in[0,1]$. Then for any $\mathfrak{t} \in \mathfrak{P}$,

$$
\begin{aligned}
{\left[\lambda \phi_{1}\right.} & \left.+(1-\lambda) \phi_{2}\right](\mathfrak{t}) \\
= & \frac{1}{\Gamma\left(r_{1}\right)} \int_{0}^{\mathfrak{t}}(\mathfrak{t}-\varsigma)^{r_{1}-1}\left[\lambda \tilde{\nu}_{1}(\varsigma)+(1-\lambda) \tilde{v}_{2}(\varsigma)\right] d \varsigma \\
& +\frac{\mathfrak{t}^{\mathfrak{v}-1}}{\Lambda}\left(\sum_{i=1}^{m} \theta_{i} \frac{\gamma_{i} \delta_{i}^{-\gamma_{i}\left(\xi_{i}+\eta_{i}\right)}}{\Gamma\left(\xi_{i}\right) \Gamma\left(r_{1}\right)}\right. \\
& \times \int_{0}^{\delta_{i}} \frac{\varsigma^{\gamma_{i}+\eta_{i}+\gamma_{i}-1}}{\left(\delta_{i}^{\gamma_{i}}-\varsigma^{\left.\gamma_{i}\right)}\right.}\left(\int_{0}^{\varsigma}(\varsigma-\sigma)^{r_{1}-1}\left[\lambda \tilde{\nu}_{1}(\sigma)+(1-\lambda) \tilde{v}_{2}(\sigma)\right] d \sigma\right) d \varsigma \\
& \left.-\frac{1}{\Gamma\left(r_{1}\right)} \int_{0}^{\mathcal{T}}(\mathcal{T}-\varsigma)^{r_{1}-1}\left[\lambda \tilde{\nu}_{1}(\varsigma)+(1-\lambda) \tilde{v}_{2}(\varsigma)\right] d \varsigma\right) .
\end{aligned}
$$

Since $Q$ has convex values, $\mathcal{R}_{Q, v}$ is convex and $\left[\lambda \tilde{v}_{1}(\mathfrak{t})+(1-\lambda) \tilde{v}_{2}(\mathfrak{t})\right] \in \mathcal{R}_{Q, v}$. Thus, $\lambda \phi_{1}+$ $(1-\lambda) \phi_{2} \in \tilde{\mathcal{S}}(v)$.

Step 2. $\tilde{\mathcal{S}}$ is bounded on bounded sets of $\mathcal{C}$.

For a constant $r>0$, let $B_{r}=\{v \in \mathcal{C}:\|v\| \leq r\}$ be a bounded set in $\mathcal{C}$. Then for each $\phi \in \tilde{\mathcal{S}}(v)$ and $v \in B_{r}$, there exists $\tilde{v} \in \mathcal{R}_{Q, v}$ such that

$$
\begin{aligned}
\phi(\mathfrak{t})= & \frac{1}{\Gamma\left(r_{1}\right)} \int_{0}^{\mathfrak{t}}(\mathfrak{t}-\varsigma)^{r_{1}-1} \tilde{v}(\varsigma) d \varsigma \\
& +\frac{\mathfrak{t}^{\mathfrak{v}-1}}{\Lambda}\left(\sum_{i=1}^{m} \theta_{i} \frac{\gamma_{i} \delta_{i}^{-\gamma_{i}\left(\xi_{i}+\eta_{i}\right)}}{\Gamma\left(\xi_{i}\right) \Gamma\left(r_{1}\right)} \int_{0}^{\delta_{i}} \frac{\varsigma^{\gamma_{i}+\eta_{i}+\gamma_{i}-1}}{\left(\delta_{i}^{\gamma_{i}}-\varsigma^{\left.\gamma_{i}\right)}\right.}\left(\int_{0}^{\varsigma}(\varsigma-\sigma)^{r_{1}-1} \tilde{v}(\sigma) d \sigma\right) d \varsigma\right. \\
& \left.-\frac{1}{\Gamma\left(r_{1}\right)} \int_{0}^{\mathcal{T}}(\mathcal{T}-\varsigma)^{r_{1}-1} \tilde{v}(\varsigma) d \varsigma\right) .
\end{aligned}
$$

Under the hypothesis (As2) and for any $\mathfrak{t} \in \mathfrak{P}$, we obtain

$$
\begin{aligned}
|\phi(\mathfrak{t})| \leq & \frac{1}{\Gamma\left(r_{1}\right)} \int_{0}^{\mathfrak{t}}(\mathfrak{t}-\varsigma)^{r_{1}-1}|\tilde{v}(\varsigma)| d \varsigma \\
& +\frac{\mathfrak{t}^{\mathfrak{v}-1}}{|\Lambda|}\left(\sum_{i=1}^{m}\left|\theta_{i}\right| \frac{\gamma_{i} \delta_{i}^{-\gamma_{i}\left(\xi_{i}+\eta_{i}\right)}}{\Gamma\left(\xi_{i}\right) \Gamma\left(r_{1}\right)} \int_{0}^{\delta_{i}} \frac{\varsigma^{\gamma_{i}+\eta_{i}+\gamma_{i}-1}}{\left(\delta_{i}^{\gamma_{i}}-\varsigma^{\gamma_{i}}\right)}\left(\int_{0}^{\varsigma}(\varsigma-\sigma)^{r_{1}-1}|\tilde{v}(\sigma)| d \sigma\right) d \varsigma\right.
\end{aligned}
$$




$$
\begin{aligned}
& \left.+\frac{1}{\Gamma\left(r_{1}\right)} \int_{0}^{\mathcal{T}}(\mathcal{T}-\varsigma)^{r_{1}-1}|\tilde{v}(\varsigma)| d \varsigma\right) \\
\leq & \frac{\|P\| \vartheta(r)}{\Gamma\left(r_{1}+1\right)}\left(\mathcal{T}^{r_{1}}+\frac{\mathcal{T}^{\mathfrak{v}+r_{1}-1}}{|\Lambda|}+\frac{\mathcal{T}^{\mathfrak{v}-1}}{|\Lambda|}\left(\sum_{i=1}^{m}\left|\theta_{i}\right| \frac{\delta_{i}^{r_{1}} \Gamma\left(\eta_{i}+\frac{r_{1}}{\gamma_{i}}+1\right)}{\Gamma\left(\eta_{i}+\frac{r_{1}}{\gamma_{i}}+\xi_{i}+1\right)}\right)\right) .
\end{aligned}
$$

Thus

$$
\|\phi\| \leq \varrho\|P\| \vartheta(r)
$$

Step 3. $\tilde{\mathcal{S}}$ sends bounded sets of $\mathcal{C}$ into equicontinuous sets.

Let $v \in B_{r}$ and $\phi \in \tilde{\mathcal{S}}(v)$. Then there is a function $\tilde{v} \in \mathcal{R}_{Q, v}$ such that

$$
\begin{aligned}
\phi(\mathfrak{t})= & \frac{1}{\Gamma\left(r_{1}\right)} \int_{0}^{\mathfrak{t}}(\mathfrak{t}-\varsigma)^{r_{1}-1} \tilde{v}(\varsigma) d \varsigma \\
& +\frac{\mathfrak{t}^{\mathfrak{v}-1}}{\Lambda}\left(\sum_{i=1}^{m} \theta_{i} \frac{\gamma_{i} \delta_{i}^{-\gamma_{i}\left(\xi_{i}+\eta_{i}\right)}}{\Gamma\left(\xi_{i}\right) \Gamma\left(r_{1}\right)} \int_{0}^{\delta_{i}} \frac{\varsigma^{\gamma_{i}+\eta_{i}+\gamma_{i}-1}}{\left(\delta_{i}^{\gamma_{i}}-\varsigma^{\gamma_{i}}\right)}\left(\int_{0}^{\varsigma}(\varsigma-\sigma)^{r_{1}-1} \tilde{v}(\sigma) d \sigma\right) d \varsigma\right. \\
& \left.-\frac{1}{\Gamma\left(r_{1}\right)} \int_{0}^{\mathcal{T}}(\mathcal{T}-\varsigma)^{r_{1}-1} \tilde{v}(\varsigma) d \varsigma\right) .
\end{aligned}
$$

Let $\mathfrak{t}_{1}, \mathfrak{t}_{2} \in \mathfrak{P}, \mathfrak{t}_{1}<\mathfrak{t}_{2}$. Then

$$
\begin{aligned}
\mid \phi\left(\mathfrak{t}_{2}\right) & -\phi\left(\mathfrak{t}_{1}\right) \mid \\
\leq & \frac{1}{\Gamma\left(r_{1}\right)} \int_{0}^{\mathfrak{t}_{1}}\left[\left(\mathfrak{t}_{2}-\varsigma\right)^{r_{1}-1}-\left(\mathfrak{t}_{1}-\varsigma\right)^{r_{1}-1}\right]|\tilde{v}(\varsigma)| d \varsigma \\
& +\frac{1}{\Gamma\left(r_{1}\right)} \int_{\mathfrak{t}_{1}}^{\mathfrak{t}_{2}}\left(\mathfrak{t}_{2}-\varsigma\right)^{r_{1}-1}|\tilde{v}(\varsigma)| d \varsigma \\
& \times \frac{\left(\mathfrak{t}_{2}^{\mathfrak{v}-1}-\mathfrak{t}_{1}^{\mathfrak{v}-1}\right)}{|\Lambda|}\left(\sum_{i=1}^{m}\left|\theta_{i}\right| \frac{\gamma_{i} \delta_{i}^{-\gamma_{i}\left(\xi_{i}+\eta_{i}\right)}}{\Gamma\left(\xi_{i}\right) \Gamma\left(r_{1}\right)} \int_{0}^{\delta_{i}} \frac{\varsigma^{\gamma_{i}+\eta_{i}+\gamma_{i}-1}}{\left(\delta_{i}^{\gamma_{i}}-\varsigma^{\gamma_{i}}\right)}\left(\int_{0}^{\varsigma}(\varsigma-\sigma)^{r_{1}-1}|\tilde{v}(\sigma)| d \sigma\right) d \varsigma\right. \\
& \left.+\frac{1}{\Gamma\left(r_{1}\right)} \int_{0}^{\mathcal{T}}(\mathcal{T}-\varsigma)^{r_{1}-1} \tilde{v}(\varsigma) d \varsigma\right) \\
\leq & \frac{\|P\| \vartheta(r)}{\Gamma\left(r_{1}+1\right)}\left(\left(\mathfrak{t}_{2}^{r_{1}}-\mathfrak{t}_{1}^{r_{1}}\right)+\frac{\left(\mathfrak{t}_{2}^{\mathfrak{v}-1}-\mathfrak{t}_{1}^{\mathfrak{v}-1}\right)}{|\Lambda|}\left(\sum_{i=1}^{m}\left|\theta_{i}\right| \frac{\delta_{i}^{r_{1}} \Gamma\left(\eta_{i}+\frac{r_{1}}{\gamma_{i}}+1\right)}{\Gamma\left(\eta_{i}+\frac{r_{1}}{\gamma_{i}}+\xi_{i}+1\right)}+\mathcal{T}^{r_{1}}\right)\right) .
\end{aligned}
$$

As $\mathfrak{t}_{1} \rightarrow \mathfrak{t}_{2}$, we obtain

$$
\left|\phi\left(\mathfrak{t}_{2}\right)-\phi\left(\mathfrak{t}_{1}\right)\right| \rightarrow 0
$$

Hence $\tilde{\mathcal{S}}\left(B_{r}\right)$ is equicontinuous. From the above-mentioned steps 2-3, along with ArzelaAscoli theorem, we infer that $\tilde{\mathcal{S}}$ is completely continuous.

Step 4. We prove that the graph of $\tilde{\mathcal{S}}$ is closed. 
Let $v_{n} \rightarrow v_{*}, \phi_{n} \in \tilde{\mathcal{S}}\left(v_{n}\right)$ and $\phi_{n}$ tends to $\phi_{*}$. We show that $\phi_{*} \in \tilde{\mathcal{S}}\left(v_{*}\right)$. Since $\phi_{n} \in \tilde{\mathcal{S}}\left(v_{n}\right)$, there exists $\tilde{v}_{n} \in \mathcal{R}_{Q, v_{n}}$ such that

$$
\begin{aligned}
\phi_{n}(\mathfrak{t})= & \frac{1}{\Gamma\left(r_{1}\right)} \int_{0}^{\mathfrak{t}}(\mathfrak{t}-\varsigma)^{r_{1}-1} \tilde{v}_{n}(\varsigma) d \varsigma \\
& +\frac{\mathfrak{t}^{\mathfrak{v}-1}}{\Lambda}\left(\sum_{i=1}^{m} \theta_{i} \frac{\gamma_{i} \delta_{i}^{-\gamma_{i}\left(\xi_{i}+\eta_{i}\right)}}{\Gamma\left(\xi_{i}\right) \Gamma\left(r_{1}\right)} \int_{0}^{\delta_{i}} \frac{\varsigma^{\gamma_{i}+\eta_{i}+\gamma_{i}-1}}{\left(\delta_{i}^{\gamma_{i}}-\varsigma^{\left.\gamma_{i}\right)}\right.}\left(\int_{0}^{\varsigma}(\varsigma-\sigma)^{r_{1}-1} \tilde{v}_{n}(\sigma) d \sigma\right) d \varsigma\right. \\
& \left.-\frac{1}{\Gamma\left(r_{1}\right)} \int_{0}^{\mathcal{T}}(\mathcal{T}-\varsigma)^{r_{1}-1} \tilde{v}_{n}(\varsigma) d \varsigma\right), \quad \mathfrak{t} \in \mathfrak{P} .
\end{aligned}
$$

Therefore, we have to prove that there exists $\tilde{v}_{*} \in \mathcal{R}_{Q, v_{*}}$ such that, for each $\mathfrak{t} \in \mathfrak{P}$,

$$
\begin{aligned}
\phi_{*}(\mathfrak{t})= & \frac{1}{\Gamma\left(r_{1}\right)} \int_{0}^{\mathfrak{t}}(\mathfrak{t}-\varsigma)^{r_{1}-1} \tilde{\boldsymbol{v}}_{*}(\varsigma) d \varsigma \\
& +\frac{\mathfrak{t}^{\mathfrak{v}-1}}{\Lambda}\left(\sum_{i=1}^{m} \theta_{i} \frac{\gamma_{i} \delta_{i}^{-\gamma_{i}\left(\xi_{i}+\eta_{i}\right)}}{\Gamma\left(\xi_{i}\right) \Gamma\left(r_{1}\right)} \int_{0}^{\delta_{i}} \frac{\varsigma^{\gamma_{i}+\eta_{i}+\gamma_{i}-1}}{\left(\delta_{i}^{\gamma_{i}}-\varsigma^{\left.\gamma_{i}\right)}\right.}\left(\int_{0}^{\varsigma}(\varsigma-\sigma)^{r_{1}-1} \tilde{\boldsymbol{v}}_{*}(\sigma) d \sigma\right) d \varsigma\right. \\
& \left.-\frac{1}{\Gamma\left(r_{1}\right)} \int_{0}^{\mathcal{T}}(\mathcal{T}-\varsigma)^{r_{1}-1} \tilde{\boldsymbol{v}}_{*}(\varsigma) d \varsigma\right) .
\end{aligned}
$$

Define the continuous linear operator $\mathcal{Z}: L^{1}(\mathfrak{P}, v) \rightarrow C(\mathfrak{P}, v)$ as follows:

$$
\begin{aligned}
\tilde{v} \rightarrow \mathcal{Z}(\tilde{v})(\mathfrak{t})= & \frac{1}{\Gamma\left(r_{1}\right)} \int_{0}^{\mathfrak{t}}(\mathfrak{t}-\varsigma)^{r_{1}-1} \tilde{v}(\varsigma) d \varsigma \\
& +\frac{\mathfrak{t}^{\mathfrak{v}-1}}{\Lambda}\left(\sum_{i=1}^{m} \theta_{i} \frac{\gamma_{i} \delta_{i}^{-\gamma_{i}\left(\xi_{i}+\eta_{i}\right)}}{\Gamma\left(\xi_{i}\right) \Gamma\left(r_{1}\right)} \int_{0}^{\delta_{i}} \frac{\varsigma^{\gamma_{i}+\eta_{i}+\gamma_{i}-1}}{\left(\delta_{i}^{\gamma_{i}}-\varsigma^{\left.\gamma_{i}\right)}\right.}\left(\int_{0}^{\varsigma}(\varsigma-\sigma)^{r_{1}-1} \tilde{v}(\sigma) d \sigma\right) d \varsigma\right. \\
& \left.-\frac{1}{\Gamma\left(r_{1}\right)} \int_{0}^{\mathcal{T}}(\mathcal{T}-\varsigma)^{r_{1}-1} \tilde{v}(\varsigma) d \varsigma\right), \quad \mathfrak{t} \in \mathfrak{P} .
\end{aligned}
$$

Notice that

$$
\begin{aligned}
& \left\|\phi_{n}-\phi_{*}\right\| \\
& =\| \frac{1}{\Gamma\left(r_{1}\right)} \int_{0}^{\mathfrak{t}}(\mathfrak{t}-\varsigma)^{r_{1}-1}\left(\tilde{v}_{n}(\varsigma)-\tilde{v}_{*}(\varsigma)\right) d \varsigma \\
& \quad+\frac{\mathfrak{t}^{\mathfrak{v}-1}}{\Lambda}\left(\sum_{i=1}^{m} \theta_{i} \frac{\gamma_{i} \delta_{i}^{-\gamma_{i}\left(\xi_{i}+\eta_{i}\right)}}{\Gamma\left(\xi_{i}\right) \Gamma\left(r_{1}\right)} \int_{0}^{\delta_{i}} \frac{\varsigma^{\gamma_{i}+\eta_{i}+\gamma_{i}-1}}{\left(\delta_{i}^{\gamma_{i}}-\varsigma^{\left.\gamma_{i}\right)}\right.}\left(\int_{0}^{\varsigma}(\varsigma-\sigma)^{r_{1}-1}\left(\tilde{v}_{n}(\sigma)-\tilde{v}_{*}(\sigma)\right) d \sigma\right) d \varsigma\right. \\
& \left.\quad-\frac{1}{\Gamma\left(r_{1}\right)} \int_{0}^{\mathcal{T}}(\mathcal{T}-\varsigma)^{r_{1}-1}\left(\tilde{v}_{n}(\varsigma)-\tilde{v}_{*}(\varsigma)\right) d \varsigma d \varsigma\right) \| \rightarrow 0,
\end{aligned}
$$

when $n \rightarrow \infty$. So in view of Lemma 6 , the operator $\mathcal{Z} \circ \mathcal{R}_{Q, v}$ has a closed graph. Moreover, we have

$$
\phi_{n} \in \mathcal{Z}\left(\mathcal{R}_{Q, v_{n}}\right) .
$$


Since $v_{n} \rightarrow v_{*}$, Lemma 6 gives

$$
\begin{aligned}
\phi_{*}(\mathfrak{t})= & \frac{1}{\Gamma\left(r_{1}\right)} \int_{0}^{\mathfrak{t}}(\mathfrak{t}-\varsigma)^{r_{1}-1} \tilde{\boldsymbol{v}}_{*}(\varsigma) d \varsigma \\
& +\frac{\mathfrak{t}^{\mathfrak{v}-1}}{\Lambda}\left(\sum_{i=1}^{m} \theta_{i} \frac{\gamma_{i} \delta_{i}^{-\gamma_{i}\left(\xi_{i}+\eta_{i}\right)}}{\Gamma\left(\xi_{i}\right) \Gamma\left(r_{1}\right)} \int_{0}^{\delta_{i}} \frac{\varsigma^{\gamma_{i}+\eta_{i}+\gamma_{i}-1}}{\left(\delta_{i}^{\gamma_{i}}-\varsigma^{\left.\gamma_{i}\right)}\right.}\left(\int_{0}^{\varsigma}(\varsigma-\sigma)^{r_{1}-1} \tilde{\boldsymbol{v}}_{*}(\sigma) d \sigma\right) d \varsigma\right. \\
& \left.-\frac{1}{\Gamma\left(r_{1}\right)} \int_{0}^{\mathcal{T}}(\mathcal{T}-\varsigma)^{r_{1}-1} \tilde{\boldsymbol{v}}_{*}(\varsigma) d \varsigma\right),
\end{aligned}
$$

for some $\tilde{v}_{*} \in \mathcal{R}_{Q, v_{*}}$.

Step 5. We show there exists an open set $\mathcal{D} \subseteq \mathcal{C}$ with $v \notin \mu \tilde{\mathcal{S}}(v)$ for each $0<\mu<1$ and $\forall v \in \partial \mathcal{D}$.

Let $\mu \in(0,1)$ and $v \in \mu \tilde{\mathcal{S}}(v)$. Then there exists $\tilde{v} \in \mathcal{R}_{Q, v}$ such that

$$
\begin{aligned}
|v(\mathfrak{t})|= & \mid \frac{\mu}{\Gamma\left(r_{1}\right)} \int_{0}^{\mathfrak{t}}(\mathfrak{t}-\varsigma)^{r_{1}-1} \tilde{v}(\varsigma) d \varsigma \\
& +\frac{\mu \mathfrak{t}^{\mathfrak{v}-1}}{\Lambda}\left(\sum_{i=1}^{m} \theta_{i} \frac{\gamma_{i} \delta_{i}^{-\gamma_{i}\left(\xi_{i}+\eta_{i}\right)}}{\Gamma\left(\xi_{i}\right) \Gamma\left(r_{1}\right)} \int_{0}^{\delta_{i}} \frac{\varsigma^{\gamma_{i}+\eta_{i}+\gamma_{i}-1}}{\left(\delta_{i}^{\gamma_{i}}-\varsigma^{\gamma_{i}}\right)}\left(\int_{0}^{\varsigma}(\varsigma-\sigma)^{r_{1}-1} \tilde{v}(\sigma) d \sigma\right) d \varsigma\right. \\
& \left.-\frac{1}{\Gamma\left(r_{1}\right)} \int_{0}^{\mathcal{T}}(\mathcal{T}-\varsigma)^{r_{1}-1} \tilde{v}(\varsigma) d \varsigma\right) \mid \\
\leq & \varrho P \| \vartheta(\|v\|) .
\end{aligned}
$$

Thus, we have

$$
|v(\mathfrak{t})| \leq \varrho\|P\| \vartheta(\|v\|), \quad \forall \mathfrak{t} \in \mathfrak{P} .
$$

Consequently, we obtain

$$
\frac{\|v\|}{\varrho\|P\| \vartheta(\|v\|)} \leq 1
$$

Under the hypothesis (As3), there is an $\mathcal{L}>0$ such that $\|v\| \neq \mathcal{L}$. We build the set $\mathcal{D}$ as follows:

$$
\mathcal{D}=\{v \in \mathcal{C}:\|v\|<\mathcal{L}\}
$$

From steps $1-4$, the operator $\tilde{\mathcal{S}}: \overline{\mathcal{D}} \rightarrow \mathcal{O}(\mathcal{C})$ is u.s.c. and completely continuous. From the choice of $\mathcal{D}$, there is no $v \in \partial \mathcal{D}$ such that $v \in \mu \tilde{\mathcal{S}}(v)$ for some $\mu \in(0,1)$. So, by LeraySchauder theorem for set-valued maps, we infer that problem (1.2) has at least one solution $v \in \overline{\mathcal{D}}$.

\subsection{The Lipschitz case}

For further existence investigation of problem (1.2) in this subsection, we deal with another existence criterion under new hypotheses. In what follows, we will demonstrate that 
our desired existence of solutions in the case of nonconvex-valued right-hand side follows by Covitz and Nadler theorem [24].

Let $(v, d)$ be a metric space induced from the normed space $(v,\|\cdot\|)$. Consider $\mathcal{H}_{d}$ : $\mathcal{O}(v) \times \mathcal{O}(v) \rightarrow \mathbb{R}^{+} \cup\{\infty\}$ defined by

$$
\mathcal{H}_{d}(\tilde{A}, \tilde{B})=\max \left\{\sup _{\tilde{a} \in \tilde{A}} d(\tilde{a}, \tilde{B}), \sup _{\tilde{b} \in \tilde{B}} d(\tilde{A}, \tilde{b})\right\},
$$

where $d(\tilde{A}, \tilde{b})=\inf _{\tilde{a} \tilde{A}} d(\tilde{a}, \tilde{b})$ and $d(\tilde{a}, \tilde{B})=\inf _{\tilde{b} \in \tilde{B}} d(\tilde{a}, \tilde{b})$. Then $\left(\mathcal{O}_{b, \mathrm{cl}}(v), \mathcal{H}_{d}\right)$ is a metric space (see [36]).

Definition 7 A set-valued operator $\tilde{\mathcal{S}}: v \rightarrow \mathcal{O}_{\mathrm{cl}}(v)$ is said to be $\kappa$-Lipschitz if and only if there exists $\kappa>0$ such that

$$
\mathcal{H}_{d}(\tilde{\mathcal{S}}(v), \tilde{\mathcal{S}}(\rho)) \leq \kappa d(v, \rho) \quad \text { for any } v, \rho \in v
$$

In particular, if $\kappa<1$, the set valued operator $\tilde{\mathcal{S}}$ is a contraction.

Theorem 2 Suppose the following hypotheses are valid:

(As4) $Q: \mathfrak{P} \times \mathbb{R} \rightarrow \mathcal{O}_{\mathrm{cp}}(\mathbb{R})$ is such that $Q(\cdot, v): \mathfrak{P} \rightarrow \mathcal{O}_{\mathrm{cp}}(\mathbb{R})$ is measurable for any $v \in \mathbb{R}$,

(As5) $\mathcal{H}_{d}(Q(\mathfrak{t}, v), Q(\mathfrak{t}, \bar{v})) \leq \varpi(\mathfrak{t})|v-\bar{v}|$ for (a.e.) all $\mathfrak{t} \in \mathfrak{P}$ and $v, \bar{v} \in \mathbb{R}$ with $\varpi \in C\left(\mathfrak{P}, \mathbb{R}^{+}\right)$and $d(0, Q(\mathfrak{t}, 0)) \leq \varpi(\mathfrak{t})$ for $($ a.e. $)$ all $\mathfrak{t} \in \mathfrak{P}$.

Then, (1.2) has at least one solution on $\mathfrak{P}$ if

$$
\varrho\|\varpi\|<1,
$$

where $\varrho$ is defined in (3.1).

Proof By using the hypothesis (As4) and Theorem III.6 in [23], $Q$ has a measurable selection $\tilde{v}: \mathfrak{P} \rightarrow \mathbb{R}, \tilde{v} \in L^{1}(\mathfrak{P}, \mathbb{R})$, and so $Q$ is integrably bounded. Thus, $\mathcal{R}_{Q, v} \neq \emptyset$. Now, we show that $\tilde{\mathcal{S}}: \mathcal{C} \rightarrow \mathcal{O}(\mathcal{C})$ defined in (3.3) satisfies the hypotheses of FPT of Nadler and Covitz. To prove that $\tilde{\mathcal{S}}(v)$ is closed for any $v \in \mathcal{C}$, let $\left\{u_{n}\right\}_{n=0}^{\infty} \in \tilde{\mathcal{S}}(v)$ be such that $\mathfrak{u}_{n} \rightarrow \mathfrak{u}$ $(n \rightarrow \infty)$ in $\mathcal{C}$. Then $\mathfrak{u} \in \mathcal{C}$ and there is $\tilde{v}_{n} \in \mathcal{R}_{Q, v_{n}}$ such that

$$
\begin{aligned}
\mathfrak{u}_{n}(\mathfrak{t})= & \frac{1}{\Gamma\left(r_{1}\right)} \int_{0}^{\mathfrak{t}}(\mathfrak{t}-\varsigma)^{r_{1}-1} \tilde{v}_{n}(\varsigma) d \varsigma \\
& +\frac{\mathfrak{t}^{\mathfrak{v}-1}}{\Lambda}\left(\sum_{i=1}^{m} \theta_{i} \frac{\gamma_{i} \delta_{i}^{-\gamma_{i}\left(\xi_{i}+\eta_{i}\right)}}{\Gamma\left(\xi_{i}\right) \Gamma\left(r_{1}\right)} \int_{0}^{\delta_{i}} \frac{\varsigma^{\gamma_{i}+\eta_{i}+\gamma_{i}-1}}{\left(\delta_{i}^{\gamma_{i}}-\varsigma^{\left.\gamma_{i}\right)}\right.}\left(\int_{0}^{\varsigma}(\varsigma-\sigma)^{r_{1}-1} \tilde{v}_{n}(\sigma) d \sigma\right) d \varsigma\right. \\
& \left.-\frac{1}{\Gamma\left(r_{1}\right)} \int_{0}^{\mathcal{T}}(\mathcal{T}-\varsigma)^{r_{1}-1} \tilde{v}_{n}(\varsigma) d \varsigma\right), \quad \forall \mathfrak{t} \in \mathfrak{P} .
\end{aligned}
$$


As $Q$ has compact values, so there exists a subsequence $\tilde{v}_{n}$ converging to $\tilde{v}$ in $L^{1}(\mathfrak{P}, \mathbb{R})$.

Thus $\tilde{v} \in \mathcal{R}_{Q, v}$, and we get

$$
\begin{aligned}
\mathfrak{u}_{n}(\mathfrak{t}) \rightarrow \mathfrak{u}(\mathfrak{t})= & \frac{1}{\Gamma\left(r_{1}\right)} \int_{0}^{\mathfrak{t}}(\mathfrak{t}-\varsigma)^{r_{1}-1} \tilde{v}(\varsigma) d \varsigma \\
& +\frac{\mathfrak{t}^{\mathfrak{v}-1}}{\Lambda}\left(\sum_{i=1}^{m} \theta_{i} \frac{\gamma_{i} \delta_{i}^{-\gamma_{i}\left(\xi_{i}+\eta_{i}\right)}}{\Gamma\left(\xi_{i}\right) \Gamma\left(r_{1}\right)} \int_{0}^{\delta_{i}} \frac{\varsigma^{\gamma_{i}+\eta_{i}+\gamma_{i}-1}}{\left(\delta_{i}^{\gamma_{i}}-\varsigma^{\gamma_{i}}\right)}\left(\int_{0}^{\varsigma}(\varsigma-\sigma)^{r_{1}-1} \tilde{v}(\sigma) d \sigma\right) d \varsigma\right. \\
& \left.-\frac{1}{\Gamma\left(r_{1}\right)} \int_{0}^{\mathcal{T}}(\mathcal{T}-\varsigma)^{r_{1}-1} \tilde{v}(\varsigma) d \varsigma\right), \quad \forall \mathfrak{t} \in \mathfrak{P} .
\end{aligned}
$$

Hence $u \in \tilde{\mathcal{S}}(v)$.

Next, we prove that there is a $\vartheta \in(0,1)(\vartheta=\varrho\|\varpi\|)$ such that

$$
H_{d}(\tilde{\mathcal{S}}(v), \tilde{\mathcal{S}}(\bar{v})) \leq \vartheta\|v-\bar{v}\| \quad \text { for each } v, \bar{v} \in C(\mathfrak{P}, \mathbb{R})
$$

Let $v, \bar{v} \in C(\mathfrak{P}, \mathbb{R})$ and $\phi_{1} \in \tilde{\mathcal{S}}(v)$. Then there exists $\tilde{v}_{1}(\mathfrak{t}) \in Q(\mathfrak{t}, v(\mathfrak{t}))$ such that

$$
\begin{aligned}
\phi_{1}(\mathfrak{t})= & \frac{1}{\Gamma\left(r_{1}\right)} \int_{0}^{\mathfrak{t}}(\mathfrak{t}-\varsigma)^{r_{1}-1} \tilde{v}_{1}(\varsigma) d \varsigma \\
& +\frac{\mathfrak{t}^{\mathfrak{v}-1}}{\Lambda}\left(\sum_{i=1}^{m} \theta_{i} \frac{\gamma_{i} \delta_{i}^{-\gamma_{i}\left(\xi_{i}+\eta_{i}\right)}}{\Gamma\left(\xi_{i}\right) \Gamma\left(r_{1}\right)} \int_{0}^{\delta_{i}} \frac{\varsigma^{\gamma_{i}+\eta_{i}+\gamma_{i}-1}}{\left(\delta_{i}^{\gamma_{i}}-\varsigma^{\left.\gamma_{i}\right)}\right.}\left(\int_{0}^{\varsigma}(\varsigma-\sigma)^{r_{1}-1} \tilde{v}_{1}(\sigma) d \sigma\right) d \varsigma\right. \\
& \left.-\frac{1}{\Gamma\left(r_{1}\right)} \int_{0}^{\mathcal{T}}(\mathcal{T}-\varsigma)^{r_{1}-1} \tilde{v}_{1}(\varsigma) d \varsigma\right) .
\end{aligned}
$$

By (As5), we have

$$
\mathcal{H}_{d}(Q(\mathfrak{t}, v), Q(\mathfrak{t}, \bar{v})) \leq \varpi(\mathfrak{t})|v(\mathfrak{t})-\bar{v}(\mathfrak{t})|
$$

So, there exists $\tilde{w}(\mathfrak{t}) \in Q(\mathfrak{t}, \bar{v})$ such that

$$
\left|\tilde{v}_{1}(\mathfrak{t})-\tilde{w}\right| \leq \varpi(\mathfrak{t})|v(\mathfrak{t})-\bar{v}(\mathfrak{t})|, \quad \mathfrak{t} \in \mathfrak{P} .
$$

We construct a set-valued map $\mathcal{E}: \mathfrak{P} \rightarrow \mathcal{O}(\mathbb{R})$ as follows:

$$
\mathcal{E}(\mathfrak{t})=\left\{\tilde{w} \in \mathbb{R}:\left|\tilde{v}_{1}(\mathfrak{t})-\tilde{w}\right| \leq \varpi(\mathfrak{t})|v(\mathfrak{t})-\bar{v}(\mathfrak{t})|\right\}
$$

We see that $\tilde{v}_{1}$ and $\sigma=\varpi|v-\bar{v}|$ are measurable, therefore we can conclude that the setvalued map $\mathcal{E}(\mathfrak{t}) \cap Q(\mathfrak{t}, \bar{v})$ is measurable. Now, we choose the function $\tilde{v}_{2}(\mathfrak{t}) \in Q(\mathfrak{t}, \bar{v})$ such that

$$
\left|\tilde{v}_{1}(\mathfrak{t})-\tilde{v}_{2}(\mathfrak{t})\right| \leq \varpi(\mathfrak{t})|v(\mathfrak{t})-\bar{v}(\mathfrak{t})|, \quad \forall \mathfrak{t} \in \mathfrak{P} .
$$


We define

$$
\begin{aligned}
\phi_{2}(\mathfrak{t})= & \frac{1}{\Gamma\left(r_{1}\right)} \int_{0}^{\mathfrak{t}}(\mathfrak{t}-\varsigma)^{r_{1}-1} \tilde{v}_{2}(\varsigma) d \varsigma \\
& +\frac{\mathfrak{t}^{\mathfrak{v}-1}}{\Lambda}\left(\sum_{i=1}^{m} \theta_{i} \frac{\gamma_{i} \delta_{i}^{-\gamma_{i}\left(\xi_{i}+\eta_{i}\right)}}{\Gamma\left(\xi_{i}\right) \Gamma\left(r_{1}\right)} \int_{0}^{\delta_{i}} \frac{\varsigma^{\gamma_{i}+\eta_{i}+\gamma_{i}-1}}{\left(\delta_{i}^{\gamma_{i}}-\varsigma^{\left.\gamma_{i}\right)}\right.}\left(\int_{0}^{\varsigma}(\varsigma-\sigma)^{r_{1}-1} \tilde{v}_{2}(\sigma) d \sigma\right) d \varsigma\right. \\
& \left.-\frac{1}{\Gamma\left(r_{1}\right)} \int_{0}^{\mathcal{T}}(\mathcal{T}-\varsigma)^{r_{1}-1} \tilde{v}_{2}(\varsigma) d \varsigma\right), \quad \forall \mathfrak{t} \in \mathfrak{P} .
\end{aligned}
$$

As a result, we obtain

$$
\begin{aligned}
\mid \phi(\mathfrak{t}) & -\phi_{2}(\mathfrak{t}) \mid \\
\leq & \frac{1}{\Gamma\left(r_{1}\right)} \int_{0}^{\mathfrak{t}}(\mathfrak{t}-\varsigma)^{r_{1}-1}\left|\tilde{v}_{1}(\varsigma)-\tilde{v}_{2}(\varsigma)\right| d \varsigma \\
& +\frac{\mathcal{T}^{\mathfrak{v}-1}}{|\Lambda|}\left(\sum_{i=1}^{m}\left|\theta_{i}\right| \frac{\gamma_{i} \delta_{i}^{-\gamma_{i}\left(\xi_{i}+\eta_{i}\right)}}{\Gamma\left(\xi_{i}\right) \Gamma\left(r_{1}\right)} \int_{0}^{\delta_{i}} \frac{\varsigma^{\gamma_{i}+\eta_{i}+\gamma_{i}-1}}{\left(\delta_{i}^{\gamma_{i}}-\varsigma^{\left.\gamma_{i}\right)}\right.}\right. \\
& \times\left(\int_{0}^{\varsigma}(\varsigma-\sigma)^{r_{1}-1}\left|\tilde{v}_{1}(\sigma)-\tilde{v}_{2}(\sigma)\right| d \sigma\right) d \varsigma \\
& \left.+\frac{1}{\Gamma\left(r_{1}\right)} \int_{0}^{\mathcal{T}}(\mathcal{T}-\varsigma)^{r_{1}-1}\left|\tilde{v}_{1}(\varsigma)-\tilde{v}_{2}(\varsigma)\right| d \varsigma\right) \\
\leq & \frac{\|\varpi\|\|v-\bar{v}\|}{\Gamma\left(r_{1}+1\right)}\left(\mathcal{T}^{r_{1}}+\frac{\mathcal{T}^{\mathfrak{v}+r_{1}-1}}{|\Lambda|}+\frac{\mathcal{T}^{\mathfrak{v}-1}}{|\Lambda|}\left(\sum_{i=1}^{m}\left|\theta_{i}\right| \frac{\delta_{i}^{r_{1}} \Gamma\left(\eta_{i}+\frac{r_{1}}{\gamma_{i}}+1\right)}{\Gamma\left(\eta_{i}+\frac{r_{1}}{\gamma_{i}}+\xi_{i}+1\right)}\right)\right) .
\end{aligned}
$$

Therefore

$$
\left\|\phi_{1}-\phi_{2}\right\| \leq \varrho\|\varpi\|\|v-\bar{v}\| .
$$

Similarly, interchanging the roles of $v$ and $\bar{v}$, we get

$$
\mathcal{H}_{d}(\tilde{\mathcal{S}}(v), \tilde{\mathcal{S}}(\bar{v})) \leq \varrho\|\varpi\|\|v-\bar{v}\| .
$$

Since $\tilde{\mathcal{S}}$ is a contraction, in the light of Covitz and Nadler theorem, we infer that $\tilde{\mathcal{S}}$ has an FP $v$ which is a solution of (1.2).

\section{Examples}

Let us first consider the following FDI:

$$
\begin{cases}{ }^{H} D^{r_{1}, r_{2}} v(\mathfrak{t}) \in Q(\mathfrak{t}, v), \mathfrak{t} \in(0, \mathcal{T}), & \mathcal{T}>0 \\ v(0)=0, & v(\mathcal{T})=\sum_{i=1}^{m} \theta_{i} I_{\gamma_{i}}^{\eta_{i} ; \xi_{i}} v\left(\delta_{i}\right)\end{cases}
$$

The next examples are special cases of the FDI given by (4.1). 
Example 1 Consider the FDI given by (4.1) with $r_{1}=\frac{5}{4}, r_{2}=0, \mathcal{T}=1, \theta_{1}=\frac{1}{4}, \theta_{2}=\frac{1}{6}, \eta_{1}=\frac{1}{2}$, $\eta_{2}=\frac{5}{2}, \xi_{1}=\frac{1}{2}, \xi_{2}=\frac{3}{2}, \gamma_{1}=\frac{1}{6}, \gamma_{2}=\frac{1}{8}, \delta_{1}=\frac{1}{4}$, and $\delta_{2}=\frac{1}{2}$ Then, problem (4.1) reduces to

$$
\left\{\begin{array}{l}
{ }^{H} D^{\frac{5}{4}, 0} v(\mathfrak{t}) \in Q(\mathfrak{t}, v), \quad \mathfrak{t} \in(0,1), \\
v(0)=0, \quad v(1)=\frac{1}{4} I_{\frac{1}{6}}^{\frac{1}{2} ; \frac{1}{2}} v\left(\frac{1}{4}\right)+\frac{1}{6} I_{\frac{1}{8}}^{\frac{5}{2} ; \frac{3}{2}} v\left(\frac{1}{2}\right),
\end{array}\right.
$$

which is an FDI involving Riemann-Liouville FD. In this case $\mathfrak{v}=\frac{5}{4}$. Let $Q:[0,1] \times \mathbb{R} \rightarrow$ $\mathcal{O}(\mathbb{R})$ be a mapping such that

$$
v \rightarrow Q(\mathfrak{t}, v)=\left[\frac{1}{6\left(\mathfrak{t}^{2}+4 \exp \left(\mathfrak{t}^{3}\right)\right)} \frac{v^{2}}{\left(v^{2}+1\right)}, \frac{1}{2 \sqrt{\mathfrak{t}+9}} \frac{|v|}{|v|+1}\right] .
$$

With reference to the above, we find $\Lambda \simeq 0.88343 \neq 0$. Obviously, $Q$ satisfies hypothesis (As1) and

$$
\|Q(\mathfrak{t}, v)\|_{\mathcal{O}}=\sup \{|\rho|: \rho \in Q(\mathfrak{t}, v)\} \leq \frac{1}{2 \sqrt{\mathfrak{t}+9}}=P(\mathfrak{t}) \vartheta(\|v\|),
$$

where $\|P\|=\frac{1}{6}$ and $\vartheta(\|v\|)=1$. Thus, assumption (As2) is fulfilled, and by (As3), we get $\mathcal{L}>0.31633$.

Therefore, all the hypotheses of Theorem 1 are valid. Hence problem (4.2) with $\mathfrak{F}$ given by (4.3) has at least one solution on $[0,1]$.

Example 2 Consider the FDI given by (4.1) with $r_{1}=\frac{3}{2}, r_{2}=1, \mathcal{T}=1, \theta_{1}=\frac{1}{2}, \theta_{2}=\frac{1}{4}, \eta_{1}=\frac{1}{4}$, $\eta_{2}=\frac{3}{2}, \xi_{1}=\frac{1}{6}, \xi_{2}=\frac{3}{2}, \gamma_{1}=\frac{1}{2}, \gamma_{2}=\frac{1}{8}, \delta_{1}=\frac{1}{6}$, and $\delta_{2}=\frac{1}{4}$. Then, problem (4.1) reduces to

$$
\left\{\begin{array}{l}
{ }^{H} D^{\frac{3}{2}, 1} v(\mathfrak{t}) \in Q(\mathfrak{t}, v), \quad \mathfrak{t} \in(0,1), \\
v(0)=0, \quad v(1)=\frac{1}{2} I_{\frac{1}{2}}^{\frac{1}{4} ; \frac{1}{6}} v\left(\frac{1}{6}\right)+\frac{1}{4} I_{\frac{1}{8}}^{\frac{3}{2} ; \frac{3}{2}} v\left(\frac{1}{4}\right),
\end{array}\right.
$$

which is an FDI involving Caputo FD. In this case, $\mathfrak{v}=2$. Let $Q:[0,1] \times \mathbb{R} \rightarrow \mathcal{O}(\mathbb{R})$ be a mapping with

$$
v \rightarrow Q(\mathfrak{t}, v)=\left[\exp \left(-v^{2}\right)+\mathfrak{t}+5, \frac{|v|}{|v|+1}+\frac{\sqrt{\mathfrak{t}+1}}{2}\right] .
$$

From the above data, we find that $\Lambda \simeq 0.92823 \neq 0$. Obviously $Q$ satisfies hypothesis (As1) and

$$
\|Q(\mathfrak{t}, v)\|_{\mathcal{O}}=\sup \{|\rho|: \rho \in Q(\mathfrak{t}, v)\} \leq 7=P(\mathfrak{t}) \vartheta(\|v\|),
$$

where $\|P\|=1$ and $\vartheta(\|v\|)=7$. Thus, assumption (As2) is fulfilled, and by (As3), we get $\mathcal{L}>6.4976$.

Therefore, all the hypotheses of Theorem 1 are valid, and consequently there exists at least one solution of $(4.4)$ on $[0,1]$ with $\mathfrak{F}$ given by $(4.5)$.

Example 3 Consider the FDI given by (4.1). Take $r_{1}=\frac{5}{4}, r_{2}=\frac{1}{2}, \mathcal{T}=1, \theta_{1}=\frac{1}{4}, \theta_{2}=\frac{1}{6}$, $\eta_{1}=\frac{1}{2}, \eta_{2}=\frac{5}{2}, \xi_{1}=\frac{1}{2}, \xi_{2}=\frac{3}{2}, \gamma_{1}=\frac{1}{6}, \gamma_{2}=\frac{1}{8}, \delta_{1}=\frac{1}{4}$, and $\delta_{2}=\frac{1}{2}$. Then, problem (4.1) reduces 
to

$$
\left\{\begin{array}{l}
{ }^{H} D^{\frac{5}{4}, \frac{1}{2}} v(\mathfrak{t}) \in Q(\mathfrak{t}, v), \quad \mathfrak{t} \in(0,1), \\
v(0)=0, \quad v(1)=\frac{1}{4} I_{\frac{1}{6}}^{\frac{1}{2} ; \frac{1}{2}} v\left(\frac{1}{4}\right)+\frac{1}{6} I_{\frac{1}{8}}^{\frac{5}{2} ; \frac{3}{2}} v\left(\frac{1}{2}\right),
\end{array}\right.
$$

which is an FDI involving Hilfer FD. In this case, we have $\mathfrak{v}=\frac{13}{8}$. Let $Q:[0,1] \times \mathbb{R} \rightarrow \mathcal{O}(\mathbb{R})$ be given by

$$
v \rightarrow Q(\mathfrak{t}, v)=\left[0, \frac{\sin (v)}{\left(\exp \left(\mathfrak{t}^{2}\right)+9\right)}+\frac{1}{15}\right]
$$

From the above data, we find that $\Lambda \simeq 0.88343 \neq 0$. Clearly, $H_{d}(Q(\mathfrak{t}, v), Q(\mathfrak{t}, \bar{v})) \leq \varpi(\mathfrak{t}) \mid v-$ $\bar{v} \mid$, where $\varpi(\mathfrak{t})=\frac{1}{\exp \left(\mathfrak{t}^{2}\right)+9}$ and $d(0, Q(\mathfrak{t}, 0))=\frac{1}{15} \leq \varpi(\mathfrak{t})$ for (a.e.) all $\mathfrak{t} \in[0,1]$. Besides, we obtain $\|\varpi\|=\frac{1}{10}$, which implies $\varrho\|\varpi\| \approx 0.19<1$. Therefore all the assumptions of Theorem 2 are valid, and hence there exists at least one solution of $(4.6)$ on $[0,1]$ with $Q$ given by (4.7).

\section{Conclusions}

We have studied a class of BVPs for Hilfer FDIs with nonlocal fractional IBC. Indeed, we acquired the existence of solutions by taking into account the cases when the set-valued map has convex or nonconvex values. The Leray-Schauder FPT was applied in the case of a convex set-valued map, whereas the FPT due to Nadler and Covitz concerning setvalued contractions was used in the case of a nonconvex set-valued map. The acquired results have been well demonstrated by numerous pertinent examples. We assert that our obtained findings are novel in the framework of Hilfer FDIs with Erdélyi-Kober fractional nonlocal IBC and they greatly contribute to the existing literature on this topic.

\section{Acknowledgements}

The authors T. Abdeljawad and B. Abdalla would like to thank Prince Sultan University for funding this work through research group Nonlinear Analysis Methods in Applied Mathematics (NAMAM) group number RG-DES-2017-01-17.

\section{Funding}

Not applicable.

Availability of data and materials

Not applicable in this study.

Competing interests

The authors declare that they have no competing interests.

Authors' contributions

All the authors contributed equally to this manuscript. All authors read and approved the final manuscript.

\section{Author details}

'Department of Mathematics, Annaba University, P.O. Box 12, Annaba 23000, Algeria. 2Department of Mathematics, Hodeidah University, Al-Hodeidah, Yemen. ${ }^{3}$ Department of Mathematics and Informatics, University of Souk Ahras, P.O. Box 1553, Souk Ahras, 41000, Algeria. ${ }^{4}$ Department of Mathematics and General Sciences, Prince Sultan University, Riyadh, Saudi Arabia. ${ }^{5}$ Department of Medical Research, China Medical University, Taichung 40402, Taiwan. ${ }^{6}$ Department of Computer Science and Information Engineering, Asia University, Taichung, Taiwan.

\section{Publisher's Note}

Springer Nature remains neutral with regard to jurisdictional claims in published maps and institutional affiliations. 


\section{References}

1. Abada, N., Benchohra, M., Hammouche, H.: Existence and controllability results for nondensely defined impulsive semilinear functional differential inclusions. J. Differ. Equ. 246(10), 3834-3863 (2009)

2. Abbas, M.I.: On the controllability of Hilfer-Katugampola fractional differential equations. Acta Comment. Univ. Tartu Math. 24(2) (2020). https://doi.org/10.12697/ACUTM.2020.24.13

3. Abbas, M.I.: On the nonlinear sequential $\Psi$-Hilfer fractional differential equations. Int. J. Math. Anal. 14(2), 77-90 (2020). https://doi.org/10.12988/ijma.2020.91283

4. Abbas, M.l.: Existence results and the Ulam stability for fractional differential equations with hybrid proportional-Caputo derivatives. J. Nonlinear Funct. Anal. 2020, Article ID 48 (2020). https://doi.org/10.23952/jnfa.2020.48

5. Abbas, M.l.: On a Hilfer fractional differential equation with nonlocal Erdélyi-Kober fractional integral boundary conditions. Filomat 34(9), 3003-3014 (2020)

6. Abdo, M.S.: Further results on the existence of solutions for generalized fractional quadratic functional integral equations. J. Math. Anal. Model. 1(1), 33-46 (2020)

7. Abdo, M.S., Abdeljawad, T., Ali, S.M., Shah, K., Jarad, F.: Existence of positive solutions for weighted fractional order differential equations. Chaos Solitons Fractals 141, 110341 (2020)

8. Abdo, M.S., Abdeljawad, T., Shah, K., Jarad, F.: Study of impulsive problems under Mittag-Leffler power law. Heliyon 6(10), e05109 (2020)

9. Abdo, M.S., Ibrahim, A.G., Panchal, S.K.: State-dependent delayed sweeping process with a noncompact perturbation in Banach spaces. Acta Univ. Apulensis 54(2), 63-74 (2018)

10. Abdo, M.S., Ibrahim, A.G., Panchal, S.K.: Noncompact perturbation of nonconvex noncompact sweeping process with delay. Comment. Math. Univ. Carol. 11(2), 1-22 (2020)

11. Abdo, M.S., Panchal, S.: Fractional integro-differential equations involving $\xi$-Hilfer fractional derivative. Adv. Appl. Math. Mech. 11, 1-22 (2019)

12. Ahmad, B., Ntouyas, S.K.: Existence results for fractional differential inclusions with Erdélyi-Kober fractional integral conditions. An. Ştiinţ. Univ. 'Ovidius' Constanţa, Ser. Mat. 25(2), 5-24 (2017)

13. Aitalioubrahim, M., Sajid, S.: Higher-order boundary value problems for Caratheodory differential inclusions. Miskolc Math. Notes 9(1), 7-15 (2008)

14. Almeida, R.: A Caputo fractional derivative of a function with respect to another function. Commun. Nonlinear Sci. Numer. Simul. 44, 460-481 (2017)

15. Almeida, R.: A Gronwall inequality for a general Caputo fractional operator. Math. Inequal. Appl. 20(4), 1089-1105 (2017)

16. Ardjouni, A., Lachouri, A., Djoudi, A.: Existence and uniqueness results for nonlinear hybrid implicit Caputo-Hadamard fractional differential equations. Open J. Math. Anal. 3(2), 106-111 (2019)

17. Asawasamrit, S., Kijjathanakorn, A., Ntouyas, S.K., Tariboon, J.: Nonlocal boundary value problems for Hilfer fractional differential equations. Bull. Korean Math. Soc. 55(6), 1639-1657 (2018)

18. Atangana, A., Baleanu, D.: New fractional derivatives with nonlocal and non-singular kernel: theory and application to heat transfer model. Therm. Sci. 20(2), 763-769 (2016)

19. Baleanu, D., Machado, J.A.T., Luo, A.C.J.: Fractional Dynamics and Control. Springer, New York (2002)

20. Benchohra, M., Ouahab, A.: Initial boundary value problems for second order impulsive functional differential inclusions. Electron. J. Qual. Theory Differ. Equ. 2003, 3 (2003)

21. Caputo, M.: Linear model of dissipation whose $Q$ is almost frequency independent. II. Geophys. J. Int. 13(5), 529-539 (1967)

22. Caputo, M., Fabrizio, M.: A new definition of fractional derivative without singular kernel. Prog. Fract. Differ. Appl. 1(2), 73-85 (2015)

23. Castaing, C., Valadier, M.: Convex Analysis and Measurable Multifunctions. Lecture Notes in Mathematics, vol. 580. Springer, Berlin (1977)

24. Covitz, H., Nadler, S.B. Jr.: Multivalued contraction mappings in generalized metric spaces. Isr. J. Math. 8, 5-11 (1970)

25. Debnath, L.: Recent applications of fractional calculus to science and engineering. Int. J. Math. Math. Sci. 54 3413-3442 (2003)

26. Deimling, K.: Multivalued Differential Equations. de Gruyter, Berlin (1992)

27. Diethelm, K.: The Analysis of Fractional Differential Equations. Lecture Notes in Mathematics. Springer, Berlin (2010)

28. Górniewicz, L.: Topological Fixed Point Theory of Set-Valued Mappings. Mathematics and Its Applications. Kluwer, Dordrecht (1999)

29. Granas, A., Dugundji, J.: Fixed Point Theory. Springer, New York (2005)

30. Hadamard, J.: Essai sur l'etude des fonctions donnees par leur developpement de Taylor. J. Math. Pures Appl. 4(8), $101-186$ (1892)

31. Hilfer, R.: Applications of Fractional Calculus in Physics. World Scientific, Singapore (2000)

32. Jarad, F., Abdeljawad, T., Baleanu, D.: Caputo-type modification of the Hadamard fractional derivatives. Adv. Differ. Equ. 2012, 142 (2012)

33. Jarad, F., Ug̃urlu, E., Abdeljawad, T., Baleanu, D.: On a new class of fractional operators. Adv. Differ. Equ. 2017(1), 247 (2017)

34. Katugampola, U.N.: A new approach to generalized fractional derivatives. Bull. Math. Anal. Appl. 6(4), 1-15 (2014)

35. Kilbas, A.A., Srivastava, H.M., Trujillo, J.J.: Theory and Applications of Fractional Differential Equations. Elsevier Science B. V., Amsterdam (2006)

36. Kisielewicz, M.: Differential Inclusions and Optimal Control. Kluwer, Dordrecht (1991)

37. Lachouri, A., Ardjouni, A., Djoudi, A.: Existence and Ulam stability results for nonlinear hybrid implicit Caputo fractional differential equations. Math. Morav. 24(1), 109-122 (2020)

38. Lachouri, A., Ardjouni, A., Djoudi, A.: Positive solutions of a fractional integro-differential equation with integral boundary conditions. Commun. Optim. Theory 2020, 1 (2020)

39. Lakshmikantham, V., Leela, S., Devi, J.V.: Theory of Fractional Dynamic Systems. Cambridge Scientific Publishers, Cambridge (2009) 
40. Lasota, A., Opial, Z:: An application of the Kakutani-Ky Fan theorem in the theory of ordinary differential equations. Bull. Acad. Pol. Sci., Sér. Sci. Math. Astron. Phys. 13, 781-786 (1965)

41. Magin, R.: Fractional calculus in bioengineering. Crit. Rev. Biomed. Eng. 32, 1-104 (2004)

42. Mali, D.A., Kucche, K.D.: Nonlocal boundary value problem for generalized Hilfer implicit fractional differential equations. Math. Methods Appl. Sci. 43(15), 8608-8631 (2020)

43. Ntouyas, S.K., Tariboon, J., Sudsutad, W.: Boundary value problems for Riemann-Liouville fractional differential inclusions with nonlocal Hadamard fractional integral conditions. Mediterr. J. Math. 13(3), 939-954 (2016)

44. Ntouyas, S.K., Tariboon, J., Thaiprayoon, C.: Nonlocal boundary value problems for Riemann-Liouville fractional differential inclusions with Hadamard fractional integral boundary conditions. Taiwan. J. Math. 20(1), 91-107 (2016)

45. Podlubny, l.: Fractional Differential Equations. Academic Press, San Diego (1999)

46. Sousa, J.V.C., Oliveira, E.C.D.: On the $\xi$-Hilfer fractional derivative. Commun. Nonlinear Sci. Numer. Simul. 60, 72-91 (2018)

47. Tarasov, V.E.: Fractional Dynamics: Application of Fractional Calculus to Dynamics of Particles, Fields and Media. Springer, New York (2011)

48. Wongcharoen, A., Ntouyas, S.K., Tariboon, J.: Boundary value problems for Hilfer fractional differential inclusions with nonlocal integral boundary conditions. Mathematics 8(11), 1905 (2020)

49. Wongcharoen, A., Ntouyas, S.K., Tariboon, J.: Nonlocal boundary value problems for Hilfer type pantograph fractional differential equations and inclusions. Adv. Differ. Equ. 2020, 279 (2020)

50. Zhou, Y.: Fractional Evolution Equations and Inclusions: Analysis and Control. Elsevier, Amsterdam (2015)

\section{Submit your manuscript to a SpringerOpen ${ }^{\circ}$ journal and benefit from:}

- Convenient online submission

- Rigorous peer review

- Open access: articles freely available online

- High visibility within the field

- Retaining the copyright to your article

Submit your next manuscript at $\gg$ springeropen.com 